\title{
Theoretical Study of the Electronic Spectra of Small Molecules That Incorporate Analogues of the Copper-Cysteine Bond
}

\author{
Hainam Do and Nicholas A. Besley*
}

School of Chemistry, University of Nottingham, University Park, Nottingham, NG7 2RD, UK.

E-mail: nick.besley@nottingham.ac.uk

${ }^{*}$ To whom correspondence should be addressed 


\begin{abstract}
The copper-sulphur bond which binds cysteinate to the metal centre is a key factor in the spectroscopy of blue copper proteins. We present theoretical calculations describing the electronically excited states of small molecules, including $\mathrm{CuSH}, \mathrm{CuSCH}_{3},\left(\mathrm{CH}_{3}\right)_{2} \mathrm{SCuSH}$, (imidazole)- $\mathrm{CuSH}$ and (imidazole) $)_{2}-\mathrm{CuSH}$, derived from the active site of blue copper proteins that contain the copper-sulphur bond in order to identify small molecular systems that have electronic structure that is analogous to the active site of the proteins. Both neutral and cationic forms are studied, since these represent the reduced and oxidised forms of the protein, respectively. For $\mathrm{CuSH}$ and $\mathrm{CuSH}^{+}$, excitation energies from time-dependent density functional theory with the B97-1 exchange-correlation functional agree well with the available experimental data and multireference configuration interaction calculations. For the positive ions, the singly occupied molecular orbital is formed from an antibonding combination of a $3 \mathrm{~d}$ orbital on copper and a $3 \mathrm{p}_{\pi}$ orbital on sulphur, which is analogous to the protein. This leads several of the molecules to have qualitatively similar electronic spectra to the proteins. For the neutral molecules, changes in the nature of the low lying virtual orbitals leads the predicted electronic spectra to vary substantially between the different molecules. In particular, addition of a ligand bonded directly to copper results in the low-lying excited states observed in $\mathrm{CuSH}$ and $\mathrm{CuSCH}_{3}$ to be absent or shifted to higher energies.
\end{abstract}

\title{
Introduction
}

Blue copper proteins are involved in a number of important biological processes, primarily to facilitate electron transfer. ${ }^{1}$ For example, in photosynthesis plastocyanin accepts an electron from cytochrome $f$ in photosystem II and transfers it to chlorophyll in photosystem I. In their oxisidised form, blue copper proteins have a rich electronic structure that has been studied extensively by a variety of spectroscopic techniques, resulting in a good understanding of the electronic structure of the active site and its dependence on the geometric structure. ${ }^{2,3}$ Plastocyanin represents a classic example of a blue copper protein. The active site of plastocyanin contains a cysteinate, two 
histidine and a methionine ligand, and is illustrated in Figure 1. The formal charge of copper in the oxidised form is +2 , while the active site including ligands has an overall formal charge of +1 owing to the formal negative charge of the cysteinate ligand.

The optical absorption spectrum of plastocyanin in its oxidised form has an intense band at 770 $\mathrm{nm}(2.07 \mathrm{eV})$ with a weaker band at $600 \mathrm{~nm}(1.60 \mathrm{eV})$, which are assigned to a $\mathrm{Cys}_{\pi} \rightarrow \mathrm{Cu}$ ligand to metal charge transfer (LMCT) transition and $\mathrm{d} \rightarrow \mathrm{d}$ ligand field excitations, respectively. ${ }^{2,4}$ At higher energy a weaker feature at about $470 \mathrm{~nm}(2.65 \mathrm{eV})$ is observed and assigned to $\mathrm{Cys}_{\sigma} \rightarrow \mathrm{Cu}$ transition. ${ }^{5}$ Detailed theoretical analysis has shown that the singly occupied molecular orbital (SOMO) can be described as the antibonding combination of the $\mathrm{Cu} 3 \mathrm{~d}_{x^{2}-y^{2}}$ orbital and the $\mathrm{S}_{\mathrm{cys}} 3 \mathrm{p}_{\pi}$ orbital, where the z-axis lies along the $\mathrm{Cu}-\mathrm{S}_{\text {methionine }}$ bond, and the $\mathrm{x}$ and $\mathrm{y}$ axes lie along the $\mathrm{Cu}$ $\mathrm{N}_{\text {histidine }}$ bonds. The intense band arises from an excitation from the bonding combination of the $\mathrm{Cu} 3 \mathrm{~d}_{x^{2}-y^{2}}$ orbital and the $\mathrm{S}_{\mathrm{cys}} 3 \mathrm{p}_{\pi}$ orbital to the SOMO. ${ }^{5-7}$ Closely related blue copper sites, such as cucumber basic protein, pseudoazurin and nitrite reductase, each have a slightly distorted active site compared to plastocyanin and exhibit remarkably different spectral features, in particular an increased intensity of the higher energy band. ${ }^{4,8}$ Furthermore, recent studies have shown that a closely related protein, nitrosocyanin, adsorbs in the red. ${ }^{9}$ This results from a change in the nature of the intense transition from an excitation from a cysteine-Cu $\pi$-like orbital to a $\sigma$-like one. This demonstrates a closely-coupled relationship between geometric and electronic structure. In contrast to the wealth of studies on the oxidised form of these proteins, much less known about the electronic structure of the reduced forms. This is largely due to the fact that the $\mathrm{d}^{10}$ electronic configuration of the reduced form does not avail itself to the spectroscopic techniques used to probe the $\mathrm{d}^{9}$ configuration of the oxidised form. The electronic structure of the reduced form has been studied with photoelectron spectroscopy combined with $\mathrm{X}_{\alpha}$ molecular orbitals calculations. ${ }^{10}$ However, it would be beneficial to have comparably detailed understanding of the reduced form because in electron transfer both oxidised and reduced forms are of equal importance. 
A key aspect that determines the observed spectroscopy and subsequent properties of blue copper proteins, is the copper-sulphur bond which binds the cysteinate to the metal centre. It is clear that the spectroscopy of the active site is sensitive to the nature of the substituents, and the orientation of the sulfur orbitals with respect to the copper d orbitals. This feature can be studied through small molecular systems that contain the copper-sulfur bond. Furthermore, such studies are not limited to the oxidised form, but also have the potential to reveal information on the reduced form. Copper hydrosulphide $(\mathrm{CuSH})$ provides the simplest model of the copper-cysteinate interaction, where $\mathrm{CuSH}$ is analogous to the reduced form of the protein and $\mathrm{CuSH}^{+}$represents the oxidised form.

In recent years, there has been a number of spectroscopic studies on CuSH. ${ }^{11-13}$ In a microwave study, $\mathrm{CuSH}$ was shown to be bent with a $\mathrm{Cu}-\mathrm{S}-\mathrm{H}$ bond angle of $93^{\circ}$, which was similar to other metal hydrosulfides. ${ }^{12}$ Using laser-induced fluorescence in combination with coupled cluster theory calculations $(\operatorname{CCSD}(\mathrm{T}) / 6-311++\mathrm{G}(3 \mathrm{df}, 3 \mathrm{dp}))$, Clouthier et al. reported a band in the $470-515$ $\mathrm{nm}(2.41-2.64 \mathrm{eV})$ region which was attributed to the $\tilde{\mathrm{A}}^{1} \mathrm{~A}^{\prime \prime}-\tilde{\mathrm{X}}^{1} \mathrm{~A}^{\prime}$ band system. ${ }^{11}$ This transition corresponds to excitation from the highest occupied molecular orbital, which is an anti-bonding mixture of a d orbital on copper and a $3 p$ orbital on sulfur, to the lowest unoccupied orbital, which was found to be non-bonding orbital on copper with significant $\mathrm{Cu}-\mathrm{S}$ antibonding character. Very recently, the $\tilde{\mathrm{B}}^{1} \mathrm{~A}^{\prime}-\tilde{\mathrm{X}}^{1} \mathrm{~A}^{\prime}$ transition was studied with resonant two color two photon ionization time-of-flight mass spectrometry. ${ }^{13}$ This transition was found near $434 \mathrm{~nm}(2.86 \mathrm{eV})$. These experiments found an increase in bond length from $2.128 \AA$ to $2.174 \AA$ and $2.202 \AA$ for the $\tilde{A}$ and $\tilde{B}$ states, respectively, but the $\mathrm{Cu}-\mathrm{S}-\mathrm{H}$ bond angle does not change significantly.

There has been considerable number of theoretical studies of the structure and spectroscopy of blue copper proteins, for example see references. ${ }^{7,14-20}$ However, there has been less attention on small molecules that incorporate the copper-cysteine bond. Ryde et al. have studied the structure and reorganization energy of small models of the copper cysteine bond, including $\mathrm{CuSH}^{0 /+}$ and 
[Cu(imidazole- $\left.\mathrm{CH}_{3}\right)_{2}\left(\mathrm{SC}_{2} \mathrm{H}_{5}\left(\mathrm{CH}_{3} \mathrm{SC}_{2} \mathrm{H}_{5}\right)\right]^{0 /+}$, using a number of theoretical methods including density functional theory (DFT) with the B3LYP exchange-correlation functional, perturbation theory and coupled cluster theory. ${ }^{21}$ It was found that the DFT calculations gave reliable results with only a small basis set dependence, where the wavefunction based methods showed a greater dependence on the basis set, and it was concluded that neither the oxidised or reduced forms of the active site were significantly strained by the protein environment. These studies show that small model molecular systems have a similar geometric structure to that found in the active site of the protein. In this paper, we explore the similarity of the electronic structure of small molecular systems to the protein through calculations of the excited states and associated electronic spectra. Figure 2 shows the molecules studied, which all include analogues of the copper-cysteine bond that is present in the active site of blue copper proteins.

\section{Computational Details}

The molecular structures were optimized using B3LYP with the Stuttgart relativistic small core (SRSC) ${ }^{22}$ basis set for copper and the 6-311G** basis set other atom types. Excited state energies and associated oscillator strengths have been determined using time-dependent density functional theory (TDDFT). In linear response TDDFT, excitation energies and intensities can be determined from the eigenvalue problem

$$
\left(\begin{array}{ll}
\mathbf{A} & \mathbf{B} \\
\mathbf{B} & \mathbf{A}
\end{array}\right)\left(\begin{array}{l}
\mathbf{X} \\
\mathbf{Y}
\end{array}\right)=\omega\left(\begin{array}{cc}
\mathbf{1} & \mathbf{0} \\
\mathbf{0} & -\mathbf{1}
\end{array}\right)\left(\begin{array}{l}
\mathbf{X} \\
\mathbf{Y}
\end{array}\right)
$$

The matrices $\mathbf{A}$ and $\mathbf{B}$ are given by

$$
\begin{aligned}
& A_{i a \sigma, j b \tau}=\delta_{i j} \delta_{a b} \delta_{\sigma \tau}\left(\varepsilon_{a}-\varepsilon_{i}\right)+K_{i a \sigma, j b \tau} \\
& B_{i a \sigma, j b \tau}=K_{i a \sigma, b j \tau}
\end{aligned}
$$


where

$$
K_{i a \sigma, j b \tau}=\iint \psi_{i \sigma}^{*}\left(\mathbf{r}_{1}\right) \psi_{a \sigma}^{*}\left(\mathbf{r}_{1}\right)\left(\frac{1}{r_{12}}+\frac{\delta^{2} E_{\mathrm{XC}}}{\delta \rho_{\sigma}\left(\mathbf{r}_{1}\right) \delta \rho_{\tau}\left(\mathbf{r}_{2}\right)}\right) \psi_{j \tau}\left(\mathbf{r}_{2}\right) \psi_{b \tau}\left(\mathbf{r}_{2}\right) d \mathbf{r}_{1} d \mathbf{r}_{2}
$$

$E_{\mathrm{XC}}$ is the exchange correlation functional and $\varepsilon_{i}$ and $\varepsilon_{a}$ are the orbital energies of the Kohn-Sham orbitals $\psi_{i}$ and $\psi_{a}$. A common approximation in TDDFT is the Tamm-Dancoff approximation (TDA $)^{23}$ wherein $\mathbf{B}$ is assumed to be zero to give the simpler eigenvalue problem

$$
\mathbf{A X}=\omega \mathbf{X}
$$

TDDFT calculations have been performed with and without the TDA in conjunction with the following exchange-correlation functionals, B97-1, ${ }^{24}$ B3LYP, ${ }^{25,26}$ BLYP, ${ }^{27,28}$ CAM-B3LYP $^{29}$ and $\mathrm{EDF} 1 .{ }^{30}$

In addition to TDDFT calculations, multi-reference configuration interaction (MRCI) calculations have been performed for $\mathrm{CuSH}$ and $\mathrm{CuSH}^{+}$with reference orbitals obtained from state averaged complete active space self-consistent field calculations, wherein the roots are weighted equally. For $\mathrm{CuSH}^{+}$an active space of $6 \mathrm{a}^{\prime}$ and $3 \mathrm{a}^{\prime \prime}$ orbitals was used, this includes the high lying orbitals shown in Figure 3, excluding the unoccupied $4 \mathrm{~s}-\mathrm{S} 3 \mathrm{p}_{\sigma}$ orbital, in addition to two further occupied a' orbitals at lower energy. For $\mathrm{CuSH}$, an active space comprising the $7 \mathrm{a}^{\prime}$ and $4 \mathrm{a}^{\prime \prime}$ orbitals was used. This includes the 8 orbitals shown in Figure 3 in addition to the three virtual Rydberg $4 \mathrm{p}$ orbitals of copper. The combined SRSC basis set for copper and $6-311 \mathrm{G}^{* *}$ basis set for other atom types was used for both TDDFT and MRCI excited state calculations. Further calculations using the SRSC basis set for copper in conjunction with the 6-311++G** basis set for other atom types are also reported. All DFT and TDDFT calculations were performed using the Q-CHEM ${ }^{31}$ software package, while Molpro ${ }^{32}$ was used for the MRCI calculations. 


\title{
Results and Discussion
}

\author{
$\mathrm{CuSH}$ and $\mathrm{CuSH}^{+}$
}

The relevant molecular orbitals for the electronic spectra of $\mathrm{CuSH}$ and $\mathrm{CuSH}^{+}$are shown in Figure 3. CuSH has the electronic configuration [core $]\left(16 a^{\prime}\right)^{2}\left(17 a^{\prime}\right)^{2}\left(6 a^{\prime \prime}\right)^{2}$. For $\mathrm{CuSH}^{+}$, which represents the copper-cysteine bond in the oxidised form of the protein, the SOMO is formed from an antibonding combination of the copper $3 \mathrm{~d}_{\mathrm{yz}}$ and sulphur $3 \mathrm{p}_{\pi}$ orbitals to give the electronic configuration [core $]\left(16 \mathrm{a}^{\prime}\right)^{2}\left(17 \mathrm{a}^{\prime}\right)^{2}\left(6 \mathrm{a}^{\prime \prime}\right)^{1}$ and a $\tilde{\mathrm{X}}^{2} \mathrm{~A}^{\prime \prime}$ ground state. This orbital is very similar to the SOMOs of blue copper proteins such as plastocyanin, which are usually described in terms of the antibonding combination of the copper $3 \mathrm{~d}_{\mathrm{x}^{2}-\mathrm{y}^{2}}$ orbital and sulphur $3 \mathrm{p}_{\pi}$ orbital due to a different choice of coordinate system. The lower lying orbitals comprise the $3 \mathrm{~d}_{\mathrm{z}^{2}}, 3 \mathrm{~d}_{\mathrm{xz}}$ and $3 \mathrm{~d}_{\mathrm{xy}}$ orbitals which are localised on the copper atom, bonding and antibonding combinations of the $3 \mathrm{~d}_{\mathrm{yz}}$ and sulphur $3 \mathrm{p}_{\sigma}$ orbitals and the bonding combination of the $3 \mathrm{~d}_{\mathrm{x}^{2}-\mathrm{y}^{2}}$ and sulphur $3 \mathrm{p}_{\pi}$ orbitals.

The computed excitation energies are given in Table 1 for TDDFT and TDDFT/TDA for a selection of exchange-correlation functionals along with results from MRCI calculations. Additional results for the EDF1 functional are included in the Supporting Information. The low lying electronic excitations correspond to transitions from the occupied orbitals to the SOMO. The lowest four transitions are the ligand field excitations from the occupied d orbitals. For these excitations there is no significant difference between the values from full TDDFT and TDDFT with the TDA. The values for the standard hybrid functionals B97-1 and B3LYP are similar, and in good agreement with the values from MRCI. While for the pure GGA functionals BLYP and EDF1, the excitation energies for the ligand field excitations are too low, while for the range-corrected CAM-B3LYP functional the predicted excitation energies are too high. All methods predict that these transitions have essentially zero intensity, whereas in the spectra for the proteins a distinct ligand field band is observed. ${ }^{2}$

The two higher lying transitions correspond to excitations from the $\mathrm{d}_{\mathrm{yz}}+\mathrm{S} 3 \mathrm{p}_{\pi}$ and $\mathrm{d}_{\mathrm{x}^{2}-\mathrm{y}^{2}}+\mathrm{S} 3 \mathrm{p}_{\sigma}$ 
orbitals to the SOMO, these orbitals are analogous to the orbitals referred to as $\mathrm{Cys}_{\pi}$ and $\mathrm{Cys}_{\sigma}$ in blue copper proteins. For these two excitations TDDFT and TDDFT/TDA make qualitatively different predictions. Within the TDA, the excitation energy for the $\mathrm{d}_{\mathrm{yz}}+\mathrm{S} 3 \mathrm{p}_{\pi} \rightarrow \mathrm{SOMO}$ transition is too high, and lies above the $\mathrm{d}_{\mathrm{x}^{2}-\mathrm{y}^{2}}+\mathrm{S} 3 \mathrm{p}_{\sigma} \rightarrow \mathrm{SOMO}$ transition for the GGA functionals. This represents a failure of the TDA, since without this approximation TDDFT results are in good agreement with the MRCI calculations, particularly for the B97-1 functional. For more details on the origin of failure of the TDA, the reader is referred elsewhere. ${ }^{33}$ The calculations show that the $\mathrm{d}_{\mathrm{yz}}+\mathrm{S} 3 \mathrm{p}_{\pi} \rightarrow \mathrm{SOMO}$ transition has significant intensity with an oscillator strength $(f)$ of 0.023 with B97-1, and the $d_{x^{2}-y^{2}}+S 3 p_{\sigma} \rightarrow$ SOMO transition is much weaker with $f=0.001$. The electronic spectrum of $\mathrm{CuSH}^{+}$is surprisingly similar to that of the oxidised forms of the protein, which highlights the importance of the $\mathrm{Cu}-\mathrm{S}$ bond in the spectroscopy of the proteins. In plastocyanin the intense $\mathrm{Cys}_{\pi}$ band occurs at $2.07 \mathrm{eV}$, with the $\mathrm{Cys}_{\sigma}$ and ligand field bands at $2.76 \mathrm{eV}$ and $1.61 \mathrm{eV}$, respectively.

The electronic structure of $\mathrm{CuSH}$ corresponds to the reduced form of the protein, and excitation energies for the low-lying transitions are given in Table 2. For this system, we focus on excitations to the LUMO and only results for full TDDFT are presented. The LUMO is shown in Figure 3 and is made from a mixture of a $3 p_{\sigma}$ orbital on sulphur and the $4 \mathrm{~s}$ orbital on copper. This is consistent with the earlier work of Clouthier et al. ${ }^{11}$ The lowest energy excitation arises from excitation from the $\mathrm{d}_{\mathrm{yz}}-\mathrm{S} 3 \mathrm{p}_{\pi}$ orbital to the LUMO. Experiment shows that the band for this transition lies at 2.5 eV. ${ }^{11}$ The results from MRCI and TDDFT with the B97-1 and CAM-B3LYP functionals are in reasonable agreement with this, while the other functionals give a lower excitation energy. The

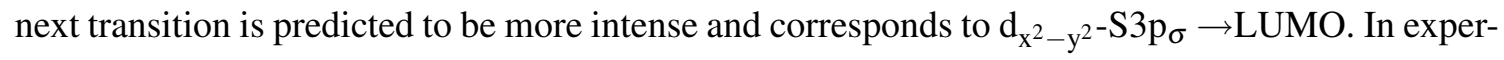
iment this transition lies at $2.86 \mathrm{eV},{ }^{13}$ and most of the calculations are consistent with this. All the theoretical methods find little separation between the transitions from the remaining $d$ orbitals that are not mixed with the orbitals of sulphur with the hybrid functionals predicting higher excitation energies than the GGA functionals and MRCI. B97-1 gives an energy for the $\mathrm{d}_{\mathrm{yz}}+\mathrm{S} 3 \mathrm{p}_{\pi} \rightarrow$ LUMO 
excitation of $3.86 \mathrm{eV}$, which is very close to the value from MRCI. The $\mathrm{d}_{\mathrm{x}^{2}-\mathrm{y}^{2}}+\mathrm{S} 3 \mathrm{p}_{\sigma} \rightarrow$ LUMO transition is significantly higher in energy $(5.07 \mathrm{eV}$ for B97-1) and is predicted to be the most intense transition. Between the transitions from the $d_{y z}+S 3 p_{\pi}$ and $d_{x^{2}-y^{2}}+S 3 p_{\sigma}$ orbitals excitations to the second virtual orbital, which is best described as a $4 \mathrm{p}$ orbital on copper, occur but these are not shown. Since these transitions involve excitation to an orbital which has more diffuse character, it is surprising that a standard hybrid functional provides accurate excitation energies. Indeed, the predicted excitation energies for the standard hybrid functionals are close to those for the range-corrected CAM-B3LYP functional. Calculation of the $\Lambda$ overlap diagnostic gives values that are low but above 0.3 , which is the value below which hybrid functionals are predicted to fail. ${ }^{34}$ Furthermore, calculations with additional diffuse basis functions (shown in Supporting Information) give results in very close agreement with those where the diffuse functions are not present. Overall, considering both cationic and neutral forms, TDDFT with the B97-1 functional gives a reliable description of the excited states, that is consistent with the available experimental data and MRCI calculations, which can be readily applied to larger molecules.

\section{Larger Molecules}

While the electronic structure of $\mathrm{CuSH}^{+}$and $\mathrm{CuSH}$ have strong similarities with the protein, significant differences are observed. In particular, for $\mathrm{CuSH}$ strong bands at low energy are observed which are not reported for the protein. Consequently, it is of interest to explore how these features evolve as further ligands are added to the copper center. Tables 3 and 4 give TDDFT/B971 excitation energies for the larger molecules, shown in Figure 2, derived from $\mathrm{CuSH}$ that contain ligands present in the active site of the protein. The molecular orbitals involved in the transitions are shown in Figures 4-7. In the gas-phase the molecular geometry can differ greatly from the structure of the active site, for example $\left(\mathrm{CH}_{3}\right)_{2} \mathrm{SCuH}$ has a $\left(\mathrm{CH}_{3}\right)_{2} \mathrm{~S}-\mathrm{Cu}$ bond length of 2.25 $\AA$ which is considerable shorter than the $\mathrm{Cu}-\mathrm{S}_{\text {methionine }}$ bond length in the protein active site and the nitrogen-copper-sulphur bond angle in (imidazole)- $\mathrm{CuSH}$ is nearly linear which is consider- 
ably different from those observed in the protein. For the positively charged ions, all of these molecules contain a SOMO that is similar to $\mathrm{CuSH}^{+}$comprising an antibonding combination of a $\mathrm{Cu} 3 \mathrm{~d}$ orbital and the sulphur $3 \mathrm{p}_{\pi}$ orbital. In addition, corresponding orbitals to the occupied orbitals involved in the electronic spectrum of $\mathrm{CuSH}^{+}$can be identified. As a result these molecules can have similar excited states and electronic spectra to $\mathrm{CuSH}^{+}$, and indeed the predicted excited states for $\mathrm{CuSCH}_{3}^{+}$and $\left(\mathrm{CH}_{3}\right)_{2} \mathrm{SCuSH}^{+}$are similar to $\mathrm{CuSH}^{+}$. For $\mathrm{CuSCH}_{3}^{+}$there is a shift to higher energy for the ligand field and $\mathrm{Cys}_{\pi}\left(\mathrm{d}_{\mathrm{xy}}+\mathrm{S} 3 \mathrm{p}_{\pi} \rightarrow \mathrm{SOMO}\right)$ excitations, while for $\left(\mathrm{CH}_{3}\right)_{2} \mathrm{SCuSH}^{+}$where there is a ligand bonded to copper there is a shift to lower energy for the ligand field and Cys $\pi$ excitations, with the energy of the $\mathrm{d}_{\mathrm{x}^{2}-\mathrm{y}^{2}}+\mathrm{S} 3 \mathrm{p}_{\sigma} \rightarrow$ SOMO transition unaffected. For $\left(\mathrm{CH}_{3}\right)_{2} \mathrm{SCuSH}^{+}$there is also an additional transition at $1.20 \mathrm{eV}$ that arises from an excitation from a p-type orbital associated with the $\mathrm{S}\left(\mathrm{CH}_{3}\right)$ group. The presence of a single imidazole group leads to a qualitatively different pattern for the excited states. The lowest energy excitation arises from the $\mathrm{d}_{\mathrm{xy}}+\mathrm{S} 3 \mathrm{p}_{\pi} \rightarrow \mathrm{SOMO}$ transition, which has no intensity. For the other molecules and the active site of the protein the corresponding transition occurs at much higher energy and has the greatest intensity. For (imidazole) $-\mathrm{CuSH}^{+}$the most intense transition is the

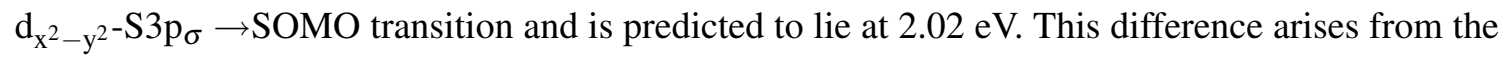
almost linear arrangement around the copper, which is very different from the structure found in the active site. Experimental spectra for different blue copper proteins show that the intensity of the $\mathrm{Cys}_{\pi}$ band is sensitive to small changes in the orientation of the ligands bonded to copper. ${ }^{2} \mathrm{On}$ the addition of a second imidazole group, the molecular structure resembles more closely that of the protein active site, and correspondingly the pattern of excited states is similar to the protein. The $\mathrm{d}_{\mathrm{xy}}+\mathrm{S} 3 \mathrm{p}_{\pi} \rightarrow \mathrm{SOMO}$ transition is the most intense and lies at $2.33 \mathrm{eV}$ with the $\mathrm{d}$ excitations predicted to lie at $\approx 1.8 \mathrm{eV}$. These energies are similar to the corresponding values of $2.07 \mathrm{eV}$ and $1.60 \mathrm{eV}$ in plastocyanin. The calculations on (imidazole) ${ }_{2}-\mathrm{CuSH}^{+}$also show a transition with significant intensity arising from an excitation from the $\pi$ orbital of the imidazole rings to the SOMO at $2.61 \mathrm{eV}$. 
For the neutral molecules, there is a greater difference between the nature of the low lying excited states for the different molecules. For $\mathrm{CuSCH}_{3}$, the substitution of hydrogen with the methyl group on the sulphur has little effect on the predicted excited states, and the computed excitation energies are similar to $\mathrm{CuSH}$. This is because the LUMO of $\mathrm{CuSH}$ is localised on the copper atom and the LUMO of $\mathrm{CuSCH}_{3}$ has a very similar form to $\mathrm{CuSH}$. However, for the other molecules which have ligands directly bonded to copper, significantly different spectra are predicted, and the low lying excitations below $3 \mathrm{eV}$ are no longer present. For the three molecules, $\left(\mathrm{CH}_{3}\right)_{2} \mathrm{SCuSH}$, (imidazole)- $\mathrm{CuSH}$ and (imidazole) $)_{2}-\mathrm{CuSH}$, the excited states arise from excitation from the two highest occupied orbitals to a range of virtual orbitals. For $\left(\mathrm{CH}_{3}\right)_{2} \mathrm{SCuSH}$ these include the $4 \mathrm{~s}$ $\mathrm{S} 3 \mathrm{p}_{\sigma}$ but this has been shifted to higher energy. Further transitions to a $\mathrm{p}$ orbital of the second sulphur atom and $4 \mathrm{p}$ orbitals of copper are also predicted. When imidazole is present, the excitations are predominantly to $\pi^{*}$ and $\sigma^{*}$ orbitals associated with the ring. For these systems, the inclusion of diffuse basis functions has a larger effect, with a lowering of the excitation energies of $0.1-0.2 \mathrm{eV}$.

\section{Conclusions}

In this work, TDDFT calculations of the electronically excited states of small molecules that contain the copper-sulphur bond present in blue copper proteins have been studied in both neutral and cationic forms to explore the similarity of their electronic structure to that of the active site of the proteins and provide a guide to future experimental studies on these systems. For CuSH and $\mathrm{CuSH}^{+}$, it is shown that excitation energies from full TDDFT calculations with the B97-1 exchange-correlation functional agree well with the available experimental data and MRCI calculations. However, for the TDA of TDDFT, qualitatively incorrect results are obtained for the $\mathrm{Cys}_{\pi}$ and $\mathrm{Cys}_{\sigma}$ excitations of $\mathrm{CuSH}^{+}$.

The SOMO of the positive ions is formed from an antibonding combination of a $3 \mathrm{~d}$ orbital 
on copper and a $3 \mathrm{p}_{\pi}$ orbital on sulphur, which is analogous to the SOMO found in proteins. The predicted electronic spectra are also found to be qualitatively similar to the proteins, except for (imidazole)-CuSH. This is because the geometry of (imidazole)-CuSH is very different from that of the protein active site. Furthermore, additional transitions with significant intensity, for example from the $\pi$ orbitals of the ring in (imidazole) ${ }_{2}-\mathrm{CuSH}$, are found. The predicted electronic spectra of the neutral molecules varies considerably, due to the changing nature of the low-lying virtual orbitals. In particular, for the molecules that contain additional ligands bonded directly to copper, the low energy excitations observed in $\mathrm{CuSH}$ and $\mathrm{CuSCH}_{3}$ are absent or shifted to higher energies.

\section{Supporting Information Available}

Calculated TDDFT excitation energies for $\mathrm{CuSH}$ and $\mathrm{CuSH}^{+}$with the EDF1 exchange-correlation functional. Calculated TDDFT excitation energies with the inclusion of diffuse basis functions. This material is available free of charge via the Internet at http://pubs.acs.org.

\section{Acknowledgments}

The authors would like to acknowledge support from the Engineering and Physical Sciences Research Council through the grant EP/I012303. The authors are grateful to Prof. Tim Wright for useful discussions.

\section{References}

(1) Gray, H.B.; Solomon, E.I., Copper Proteins; Ed: Spiro, T.G.; Wiley, New York, 1981.

(2) Solomon, E.I.; Szilagyi, R.K.; George, S.D.; Basumallick, L., Chem. Rev. 2004, 104, 419458.

(3) Solomon, E.I., Inorg. Chem. 2006, 45, 8012-8025. 
(4) LaCroix, L.B; Shadle, S.E.; Wang, Y.; Averill, B.A.; Hedman, B.; Hodgson, K.O.; Solomon, E.I., J. Am. Chem. Soc. 1996, 118, 7755-7768.

(5) Gewirth, A.A.; Solomon, E.I., J. Am. Chem. Soc. 1988, 115, 3811-3819.

(6) Penfield, K.W.; Gewirth, A.A.; Solomon, E.I., J. Am. Chem. Soc. 1985, 107, 4519-4529.

(7) Pierloot, K.; De Kerpel, J.O.A.; Ryde, U.; Roos, B.O., J. Am. Chem. Soc. 1997, 119, 218-226.

(8) LaCroix, L.B.; Randall, D.W.; Nerissian, A.M.; Hoitnik, C.W.G.; Canters, G.W.; Valentine, J.S.; Solomon, E.I., J. Am. Chem. Soc. 1998, 120, 9621-9631.

(9) Basumallick, L.; Sarangi, R.; George, S.D.; Elmore, B.; Hooper, A.B.; Hedman, B.; Hodgson, K.O.; Solomon, E.I., J. Am. Chem. Soc. 2005, 127, 3531-3544.

(10) Guckert, J.A.; Lowery, M.D.; Solomon, E.I., J. Am. Chem. Soc. 1995, 117, 2817-2844.

(11) Sunahori, F.X.; Zhang, X.; Clouthier, D.J., J. Chem. Phys. 2006, 125, 084310.

(12) Janczyk, A.; Walter, S.K.; Ziurys, L.M., Chem. Phys. Lett. 2005, 401, 211-216.

(13) Kokkin, D.L.; Reilly, N.J.; McCarthy, M.C.; Stanton, J.F., J. Mol. Spectrosc. 2011, 268, 23-27.

(14) Pierloot, K.; De Kerpel, J.O.A.; Ryde, U.; Olsson, M.H.M.; Roos, B.O., J. Am. Chem. Soc. 1998, 120, 13156-13166.

(15) Sinnecker, S.; Neese, F., J. Comput. Chem. 2006, 27, 1463-1475.

(16) Deeth, R.J., Inorg. Chem. 2007, 46, 4492-4503.

(17) Cascella, M.; Cuendet, M.A.; Tavernelli, I.; Rothlisberger, U., J. Phys. Chem. B 2007, 111, 10248-10252.

(18) Vancoillie, S.; Pierloot, K., J. Phys. Chem. A 2008, 112, 4011-4019. 
(19) Robinson, D.; Besley, N.A., Phys. Chem. Chem. Phys. 2010, 12, 9667-9676.

(20) Besley, N.A.; Robinson, D., Faraday Discussions 2011, 148, 55-70.

(21) Ryde, U.; Olsson, M.H.M.; Roos, B.O.; Borin, A.C., Theor. Chem. Acc. 2001, 105, 452-462.

(22) Bergner, A.; Dolg, M.; Kuechle, W.; Stoll, H.; Preuss, H., Mol. Phys. 1993, 80, 1431-1441.

(23) Hirata, S.; Head-Gordon, M., Chem. Phys. Lett. 1999, 314, 291-299.

(24) Hamprecht, F.A.; Cohen, A.J.; Tozer, D.J.; Handy, N.C., J. Chem. Phys. 1998, 109, 62646271.

(25) Becke, A.D., J. Chem. Phys. 1993, 98, 5648-5652.

(26) Stephens, P.J.; Devlin, F.J.; Chabalowski, C.F.; Frisch, M.J., J. Phys. Chem. 1994, 98, 1162311627.

(27) Becke, A.D., Phys. Rev. A 1988, 38, 3098-3100.

(28) Lee, C.T.; Yang, W.T.; Parr, R.G., Phys. Rev. B 1988, 37, 785-789.

(29) Yanai, T.; Tew, D.P.; Handy, N.C. Chem. Phys. Lett. 2004, 393, 51-57.

(30) Adamson, R.D.; Gill, P.M.W.; Pople, J.A., Chem. Phys. Lett. 1998, 284, 6-11.

(31) Shao, Y.; Molnar, L. F.; Jung, Y.; Kussmann, J.; Ochsenfeld, C.; Brown, S. T.; Gilbert, A. T. B.; Slipchenko, L. V.; Levchenko, S. V.; O’Neill, D. P.; DiStasio Jr, R. A.; Lochan, R. C.; Wang, T.; Beran, G. J. O.; Besley, N. A.; Herbert, J. M.; Lin, C. Y.; Voorhis, T. V.; Chien, S.-H.; Sodt, A.; Steele, R. P.; Rassolov, V. A.; Maslen, P. E.; Korambath, P. P.; Adamson, R. D.; Austin, B.; Baker, J.; Byrd, E. F. C.; Dachsel, H.; Doerksen, R. J.; Dreuw, A.; Dunietz, B. D.; Dutoi, A. D.; Furlani, T. R.; Gwaltney, S. R.; Heyden, A.; Hirata, S.; Hsu, C.-P.; Kedziora, G.; Khalliulin, R. Z.; Klunzinger, P.; Lee, A. M.; Lee, M. S.; Liang, W.; Lotan, I.; Nair, N.; Peters, B.; Proynov, E. I.; Pieniazek, P. A.; Rhee, Y. M.; Ritchie, J.; Rosta, E.; 
Sherrill, C. D.; Simmonett, A. C.; Subotnik, J. E.; Woodcock III, H. L.; Zhang, W.; Bell, A. T.; Chakraborty, A. K.; Chipman, D. M.; Keil, F. J.; Warshel, A.; Hehre, W. J.; Schaefer III, H. F.; Kong, J.; Krylov, A. I.; Gill, P. M. W.; Head-Gordon, M. Phys. Chem. Chem. Phys. 2006, 8, 3172-3191.

(32) MOLPRO, version 2006.1, a package of ab initio programs, Werner, H.-J. P.; Knowles, P.J.; Lindh, R.; Manby, F.R.; Schütz, M.; Celani, P.; Korona, T.; Rauhut, G.; Amos, R.D.; Bernhardsson, A.; Berning, A.; Cooper, D.L.; Deegan, M.J.O.; Dobbyn, A.J.; Eckert, F.; Hampel, C.; Hetzer, G.; Lloyd, A.W.; McNicholas, S.J.; Meyer, W.; Mura, M.E.; Nicklass, A.; Palmieri, P.; Pitzer, R.; Schumann, U.; Stoll, H.; Stone, A.J.; Tarroni, R.; Thorsteinsson. T.

(33) Grüning, M.; Marini, A.; Gonze, X., Nano Lett. 2009, 9, $2820-2024$.

(34) Peach, M.J.G.; Benfield, P.; Helgaker, T.; Tozer, D.J., J. Chem. Phys. 2008, 128, 044118. 
Table 1: Calculated vertical excitation energies of $\mathrm{CuSH}^{+}$in $\mathrm{eV}$. TDA - Tamm-Dancoff approximation, FULL - full TDDFT.

\begin{tabular}{|c|cc|cc|cc|cc|c|}
\hline Excitation & \multicolumn{2}{|c|}{ B97-1 } & \multicolumn{2}{|c|}{ B3LYP } & \multicolumn{2}{|c|}{ BLYP } & \multicolumn{2}{c|}{ CAM-B3LYP } & MRCI \\
& TDA & FULL & TDA & FULL & TDA & FULL & TDA & FULL & \\
\hline $\mathrm{d}_{\mathrm{x}^{2}-\mathrm{y}^{2}-\mathrm{S} 3 \mathrm{p}_{\sigma} \rightarrow \text { SOMO }}$ & 0.92 & 0.88 & 0.96 & 0.93 & 0.84 & 0.81 & 1.11 & 1.06 & 0.70 \\
$\mathrm{~d}_{\mathrm{xy}} \rightarrow$ SOMO & 1.77 & 1.74 & 1.82 & 1.80 & 1.28 & 1.27 & 2.34 & 2.32 & 1.82 \\
$\mathrm{~d}_{\mathrm{xz}} \rightarrow$ SOMO & 1.82 & 1.80 & 1.86 & 1.84 & 1.28 & 1.28 & 2.50 & 2.49 & 1.91 \\
$\mathrm{~d}_{\mathrm{z}^{2}} \rightarrow$ SOMO & 1.83 & 1.80 & 1.87 & 1.85 & 1.37 & 1.36 & 2.50 & 2.49 & 1.91 \\
$\mathrm{~d}_{\mathrm{yz}}+\mathrm{S} 3 \mathrm{p}_{\pi} \rightarrow$ SOMO & 2.64 & 2.29 & 2.71 & 2.40 & 2.92 & 2.46 & 3.01 & 2.80 & 2.30 \\
$\mathrm{~d}_{\mathrm{x}^{2}-\mathrm{y}^{2}}+\mathrm{S} 3 \mathrm{p}_{\sigma} \rightarrow$ SOMO & 2.71 & 2.65 & 2.73 & 2.68 & 2.67 & 2.63 & 3.03 & 2.99 & 2.59 \\
\hline
\end{tabular}


Table 2: Calculated vertical excitation energies of $\mathrm{CuSH}$ in eV, non-zero oscillator strengths given in parenthesis. ${ }^{\dagger}$ values from experiment, references ${ }^{11,13}$

\begin{tabular}{|c|c|c|c|c|c|c|}
\hline Excitation & B97-1 & B3LYP & BLYP & CAM-B3LYP & MRCI & Exp..$^{\dagger}$ \\
\hline $\mathrm{d}_{\mathrm{yz}}-\mathrm{S} 3 \mathrm{p}_{\pi} \rightarrow$ LUMO & $2.18(0.009)$ & $2.08(0.008)$ & $1.74(0.006)$ & $2.34(0.011)$ & 2.27 & 2.5 \\
$\mathrm{~d}_{\mathrm{x}^{2}-\mathrm{y}^{2}-\mathrm{S} 3 \mathrm{p}_{\sigma} \rightarrow \text { LUMO }}$ & $2.94(0.035)$ & $2.92(0.037)$ & $2.62(0.004)$ & $2.90(0.030)$ & 2.57 & 2.86 \\
$\mathrm{~d}_{\mathrm{xz}} \rightarrow$ LUMO & 3.19 & 3.14 & 2.64 & 3.13 & 2.90 & \\
$\mathrm{~d}_{\mathrm{z}^{2}} \rightarrow$ LUMO & $3.20(0.001)$ & $3.15(0.001)$ & $2.76(0.029)$ & $3.14(0.001)$ & 2.91 & \\
$\mathrm{~d}_{\mathrm{xy}} \rightarrow$ LUMO & $3.32(0.005)$ & $3.26(0.005)$ & $2.85(0.005)$ & $3.27(0.005)$ & 2.94 & \\
$\mathrm{~d}_{\mathrm{yz}}+\mathrm{S} 3 \mathrm{p}_{\pi} \rightarrow$ LUMO & 3.86 & 3.77 & 3.30 & $3.95(0.001)$ & 3.92 & \\
$\mathrm{~d}_{\mathrm{x}^{2}-\mathrm{y}^{2}}+\mathrm{S} 3 \mathrm{p}_{\sigma} \rightarrow$ LUMO & $5.07(0.052)$ & $5.01(0.049)$ & $4.71(0.021)$ & $5.23(0.064)$ & - & \\
\hline
\end{tabular}


Table 3: Calculated TDDFT/B97-1 excitation energies in eV for the larger molecule cations. Nonzero oscillator strengths given in parenthesis.

\begin{tabular}{|c|c|}
\hline Excitation & $\Delta \mathrm{E} / \mathrm{eV}$ \\
\hline $\mathrm{CuSCH}_{3}^{+}$ & \\
\hline 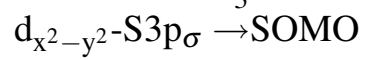 & 0.93 \\
\hline $\mathrm{d}_{\mathrm{yz}} \rightarrow$ SOMO & 2.05 \\
\hline $\mathrm{d}_{\mathrm{xz}} \rightarrow \mathrm{SOMO}$ & 2.16 \\
\hline $\mathrm{d}_{\mathrm{z}^{2}} \rightarrow \mathrm{SOMO}$ & 2.16 \\
\hline $\mathrm{d}_{\mathrm{xy}}+\mathrm{S} 3 \mathrm{p}_{\pi} \rightarrow \mathrm{SOMO}$ & $2.49(0.017)$ \\
\hline $\mathrm{d}_{\mathrm{x}^{2}-\mathrm{y}^{2}}+\mathrm{S} 3 \mathrm{p}_{\sigma} \rightarrow$ SOMO & $2.64(0.001)$ \\
\hline$\left(\mathrm{CH}_{3}\right)_{2} \mathrm{SCuSH}^{+}$ & \\
\hline $\mathrm{d}_{\mathrm{x}^{2}-\mathrm{y}^{2}}-\mathrm{S} 3 \mathrm{p}_{\sigma} \rightarrow \mathrm{SOMO}$ & 0.67 \\
\hline $\mathrm{S}^{\mathrm{CH}_{3}} 3 \mathrm{p}_{\sigma} \rightarrow \mathrm{SOMO}$ & 1.20 \\
\hline $\mathrm{d}_{\mathrm{xy}} \rightarrow \mathrm{SOMO}$ & $1.34(0.001)$ \\
\hline $\mathrm{d}_{\mathrm{xz}} \rightarrow \mathrm{SOMO}$ & 1.47 \\
\hline $\mathrm{d}_{\mathrm{z}^{2}} \rightarrow \mathrm{SOMO}$ & 1.48 \\
\hline $\mathrm{d}_{\mathrm{yz}}+\mathrm{S} 3 \mathrm{p}_{\pi} \rightarrow \mathrm{SOMO}$ & $2.05(0.070)$ \\
\hline $\mathrm{d}_{\mathrm{x}^{2}-\mathrm{y}^{2}}+\mathrm{S} 3 \mathrm{p}_{\sigma} \rightarrow \mathrm{SOMO}$ & 2.62( \\
\hline (imidazole)-CuSH ${ }^{+}$ & \\
\hline $\mathrm{d}_{\mathrm{xy}}+\mathrm{S} 3 \mathrm{p}_{\pi} \rightarrow \mathrm{SOMO}$ & 0.72 \\
\hline$\pi_{\text {ring }} \rightarrow$ SOMO & $1.27(0.010)$ \\
\hline $\mathrm{d}_{\mathrm{yz}} \rightarrow \mathrm{SOMO}$ & 1.47 \\
\hline $\mathrm{d}_{\mathrm{z}^{2}} \rightarrow \mathrm{SOMO}$ & 1.5 \\
\hline $\mathrm{d}_{\mathrm{xz}} \rightarrow \mathrm{SOMO}$ & 1.56 \\
\hline $\mathrm{d}_{\mathrm{x}^{2}-\mathrm{y}^{2}}-\mathrm{S} 3 \mathrm{p}_{\sigma} \rightarrow \mathrm{SOMO}$ & $2.02(0.090)$ \\
\hline $\mathrm{d}_{\mathrm{x}^{2}-\mathrm{y}^{2}}+\mathrm{S} 3 \mathrm{p}_{\sigma} \rightarrow$ SOMO & $2.05(0.001)$ \\
\hline$(\text { imidazole })_{2}-\mathrm{CuS}$ & \\
\hline $\mathrm{d}_{\mathrm{x}^{2}-\mathrm{y}^{2}}-\mathrm{S} 3 \mathrm{p}_{\sigma} \rightarrow \mathrm{SOMO}$ & 1.08 \\
\hline $\mathrm{d}_{\mathrm{z}^{2}} \rightarrow \mathrm{SOMO}$ & $1.77(0.001)$ \\
\hline $\mathrm{d}_{\mathrm{xz}} \rightarrow \mathrm{SOMO}$ & 1.88 \\
\hline $\mathrm{d}_{\mathrm{yz}} \rightarrow \mathrm{SOMO}$ & 1.95 \\
\hline $\mathrm{d}_{\mathrm{xy}}+\mathrm{S} 3 \mathrm{p}_{\pi} \rightarrow \mathrm{SOMO}$ & $2.33(0.095)$ \\
\hline$\pi_{1} \rightarrow \mathrm{SOMO}$ & $2.61(0.009)$ \\
\hline$\pi_{2} \rightarrow \mathrm{SOMO}$ & 2.68 \\
\hline $\mathrm{d}_{\mathrm{x}^{2}-\mathrm{y}^{2}}+\mathrm{S} 3 \mathrm{p}_{\sigma} \rightarrow \mathrm{SOMO}$ & 3.11 \\
\hline
\end{tabular}


Table 4: Calculated TDDFT/B97-1 excitation energies in $\mathrm{eV}$ for the larger molecules. Non-zero oscillator strengths given in parenthesis.

\begin{tabular}{|c|c|}
\hline Excitation & $\Delta \mathrm{E} / \mathrm{eV}$ \\
\hline $\mathrm{CuSCH}_{3}$ & \\
\hline $\mathrm{d}_{\mathrm{xy}}-\mathrm{S} 3 \mathrm{p}_{\pi} \rightarrow 4 \mathrm{~s}-\mathrm{S} 3 \mathrm{p}_{\sigma}$ & $1.92(0.006)$ \\
\hline 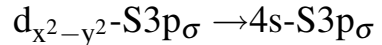 & $2.84(0.054)$ \\
\hline $\mathrm{d}_{\mathrm{z}^{2}} \rightarrow 4 \mathrm{~s}-\mathrm{S} 3 \mathrm{p}_{\sigma}$ & $3.17(0.001)$ \\
\hline $\mathrm{d}_{\mathrm{xz}} \rightarrow 4 \mathrm{~s}-\mathrm{S} 3 \mathrm{p}_{\sigma}$ & 3.17 \\
\hline $\mathrm{d}_{\mathrm{yz}} \rightarrow 4 \mathrm{~s}-\mathrm{S} 3 \mathrm{p}_{\sigma}$ & $3.28(0.005)$ \\
\hline $\mathrm{d}_{\mathrm{xy}}+\mathrm{S} 3 \mathrm{p}_{\pi} \rightarrow 4 \mathrm{~s}-\mathrm{S} 3 \mathrm{p}_{\sigma}$ & 3.73 \\
\hline $\mathrm{d}_{\mathrm{x}^{2}-\mathrm{y}^{2}}+\mathrm{S} 3 \mathrm{p}_{\sigma} \rightarrow 4 \mathrm{~s}-\mathrm{S} 3 \mathrm{p}_{\sigma}$ & $4.61(0.047)$ \\
\hline$\left(\mathrm{CH}_{3}\right)_{2} \mathrm{SCuSH}$ & \\
\hline $\mathrm{d}_{\mathrm{yz}}-\mathrm{S} 3 \mathrm{p}_{\pi} \rightarrow 4 \mathrm{~s}-\mathrm{S} 3 \mathrm{p}_{\sigma}$ & $3.95(0.024)$ \\
\hline $\mathrm{d}_{\mathrm{yz}}-\mathrm{S} 3 \mathrm{p}_{\pi} \rightarrow \mathrm{S}^{\mathrm{CH}_{3}} 3 \mathrm{p}$ & $4.03(0.050)$ \\
\hline $\mathrm{d}_{\mathrm{yz}}-\mathrm{S} 3 \mathrm{p}_{\pi} \rightarrow \mathrm{Cu} 4 \mathrm{p}_{\mathrm{x}}$ & $4.27(0.003)$ \\
\hline 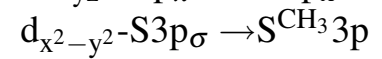 & $4.67(0.025)$ \\
\hline $\mathrm{d}_{\mathrm{x}^{2}-\mathrm{y}^{2}}-\mathrm{S} 3 \mathrm{p}_{\sigma} \rightarrow 4 \mathrm{~s}-\mathrm{S} 3 \mathrm{p}_{\sigma}$ & $4.92(0.036)$ \\
\hline 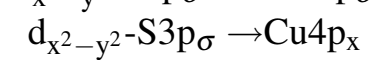 & $5.24(0.047)$ \\
\hline (imidazole)-CuSH & \\
\hline $\mathrm{d}_{\mathrm{xy}}-\mathrm{S} 3 \mathrm{p}_{\pi} \rightarrow \pi_{1}^{*}$ & $3.61(0.032)$ \\
\hline $\mathrm{d}_{\mathrm{xy}}-\mathrm{S} 3 \mathrm{p}_{\pi} \rightarrow \sigma_{\mathrm{NH}}^{*}$ & $3.85(0.018)$ \\
\hline $\mathrm{d}_{\mathrm{x}^{2}-\mathrm{y}^{2}}-\mathrm{S} 3 \mathrm{p}_{\sigma} \rightarrow \pi_{1}^{*}$ & $4.19(0.001)$ \\
\hline $\mathrm{d}_{\mathrm{xy}}-\mathrm{S} 3 \mathrm{p}_{\pi} \rightarrow \mathrm{Cu} 4 \mathrm{p}_{\mathrm{x}}$ & 4.42 \\
\hline $\mathrm{d}_{\mathrm{xy}}-\mathrm{S} 3 \mathrm{p}_{\pi} \rightarrow \pi_{2}^{*}$ & $4.47(0.017)$ \\
\hline $\mathrm{d}_{\mathrm{x}^{2}-\mathrm{y}^{2}}-\mathrm{S} 3 \mathrm{p}_{\sigma} \rightarrow \sigma_{\mathrm{NH}}^{*}$ & $4.76(0.025)$ \\
\hline (imidazole) $)_{2}-\mathrm{CuSH}$ & \\
\hline $\mathrm{d}_{\mathrm{xy}}-\mathrm{S} 3 \mathrm{p}_{\pi} \rightarrow \pi_{1}^{*}$ & $3.36(0.010)$ \\
\hline$d_{x y}-S 3 p_{\pi} \rightarrow \sigma_{N H}^{*}$ & $3.43(0.016)$ \\
\hline $\mathrm{d}_{\mathrm{xy}}-\mathrm{S} 3 \mathrm{p}_{\pi} \rightarrow \pi_{2}^{*}$ & $3.52(0.003)$ \\
\hline 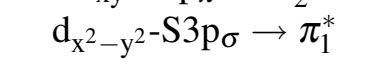 & $3.55(0.013)$ \\
\hline$d_{x^{2}-y^{2}}-S 3 p_{\sigma} \rightarrow \sigma_{N H}^{*}$ & $3.70(0.010)$ \\
\hline 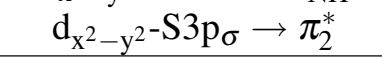 & $3.78(0.008)$ \\
\hline
\end{tabular}




\section{Figure Captions}

Figure 1: Active site of plastocyanin

Figure 2: Small molecules with the copper-cysteine bond.

Figure 3: Molecular orbitals of $\mathrm{CuSH}$.

Figure 4: Molecular orbitals of $\mathrm{CuSCH}_{3}$.

Figure 5: Molecular orbitals of $\left(\mathrm{CH}_{3}\right)_{2} \mathrm{CuSH}$.

Figure 6: Molecular orbitals of (imidazole)-CuSH.

Figure 7: Molecular orbitals of (imidazole) ${ }_{2}-\mathrm{CuSH}$. 
Figure 1

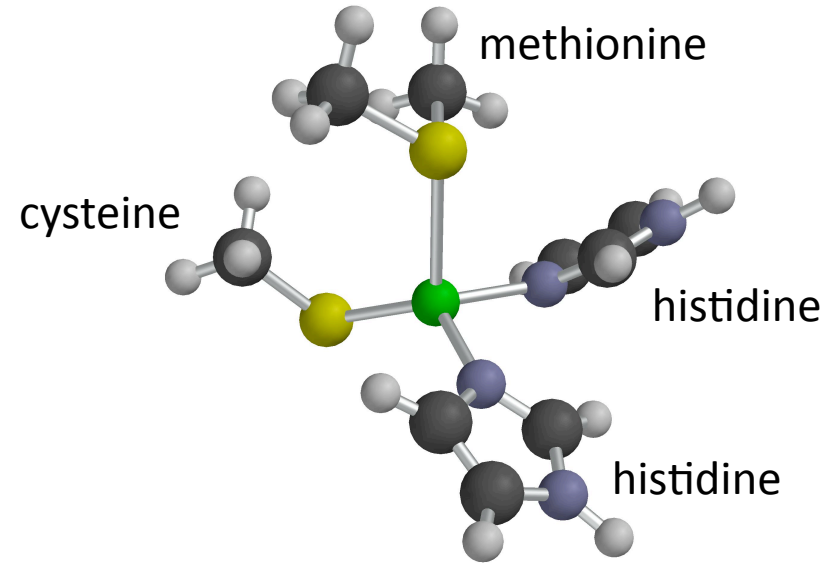


Figure 2

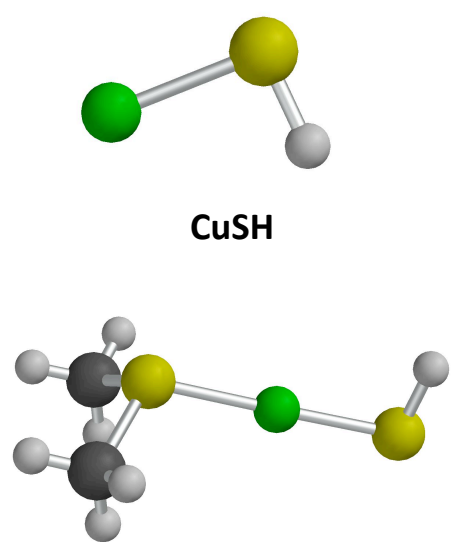

$\left(\mathrm{CH}_{3}\right)_{2} \mathrm{SCuSH}$

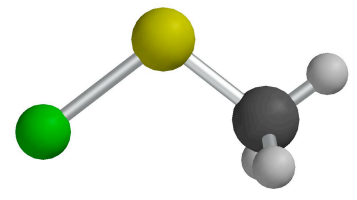

$\mathrm{CuSCH}_{3}$

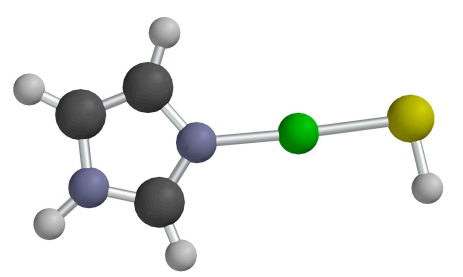

ImidazoleCuSH

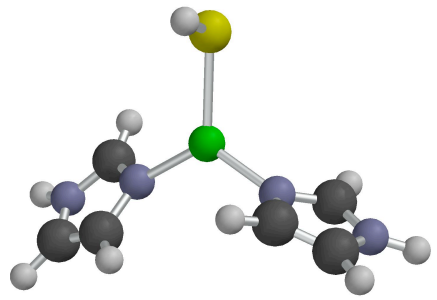

Imidazole ${ }_{2} \mathrm{CuSH}$ 
Figure 3

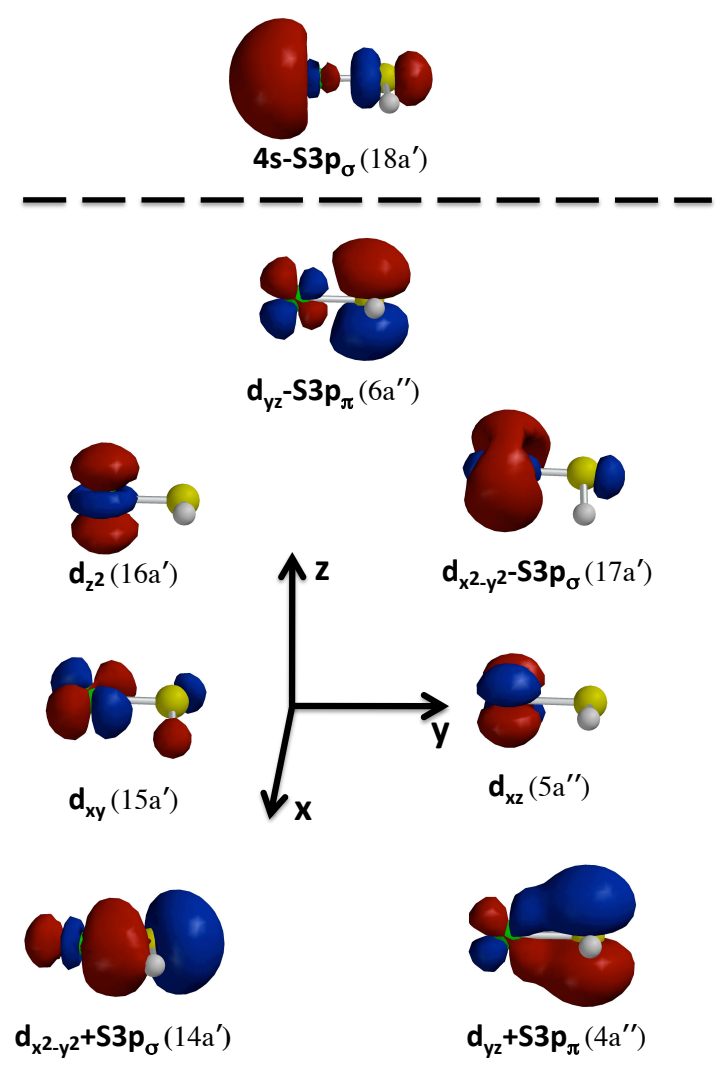


Figure 4

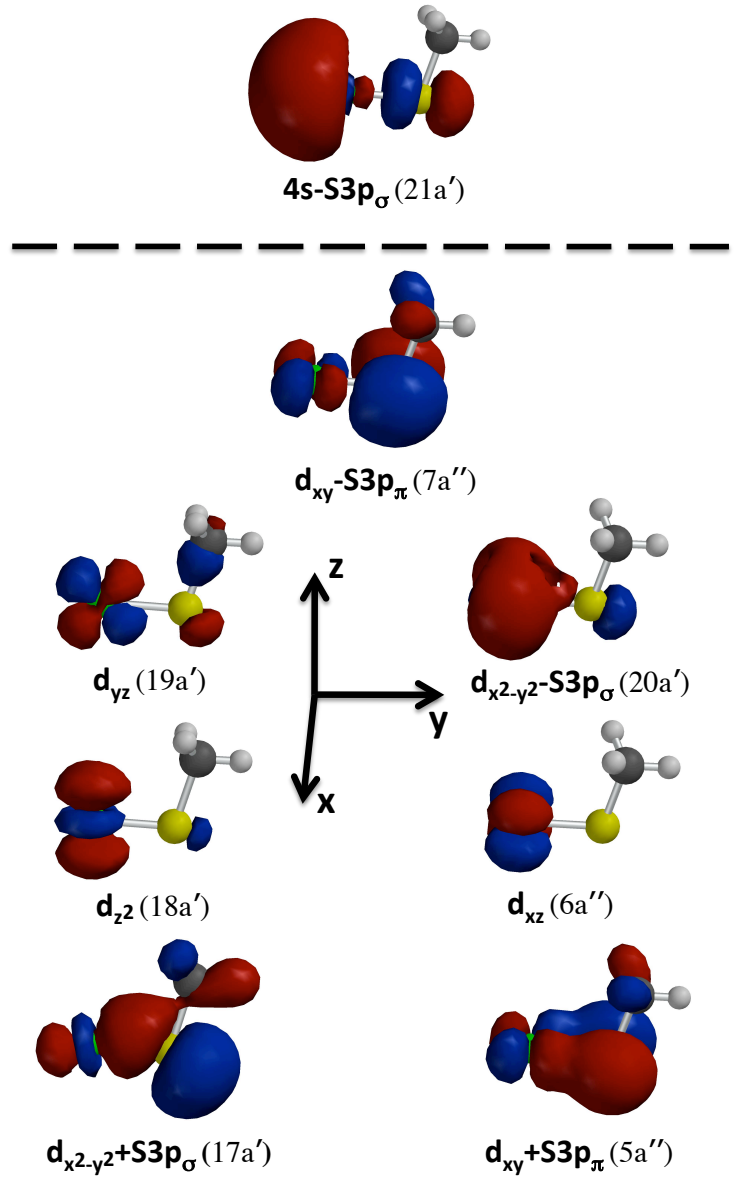


Figure 5
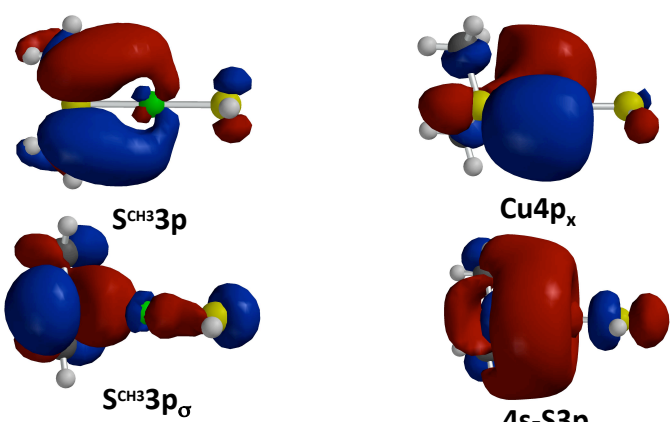

Cu4p

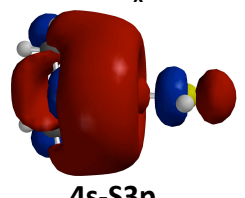

$4 s-S 3 p_{\sigma}$
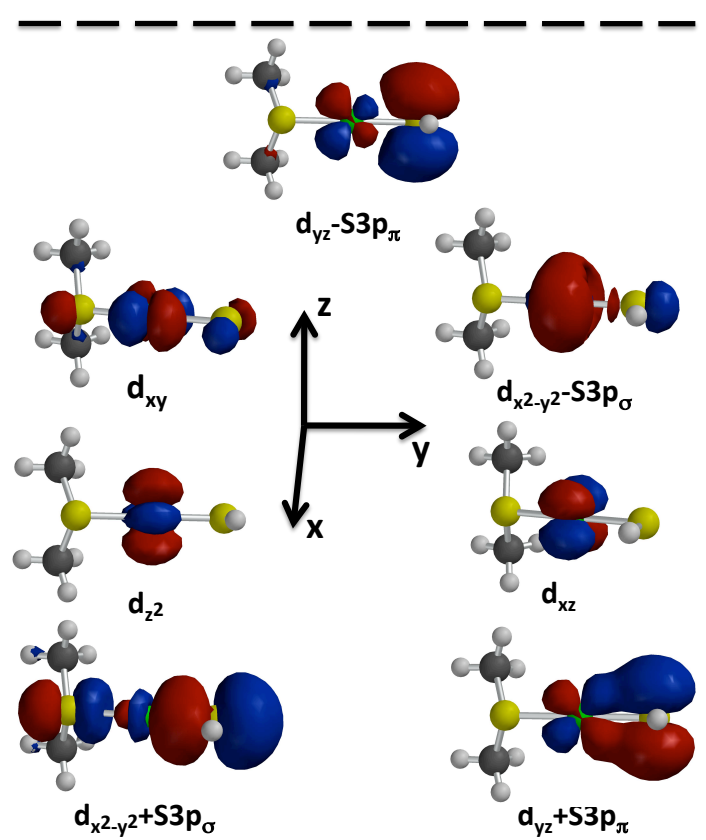
Figure 6

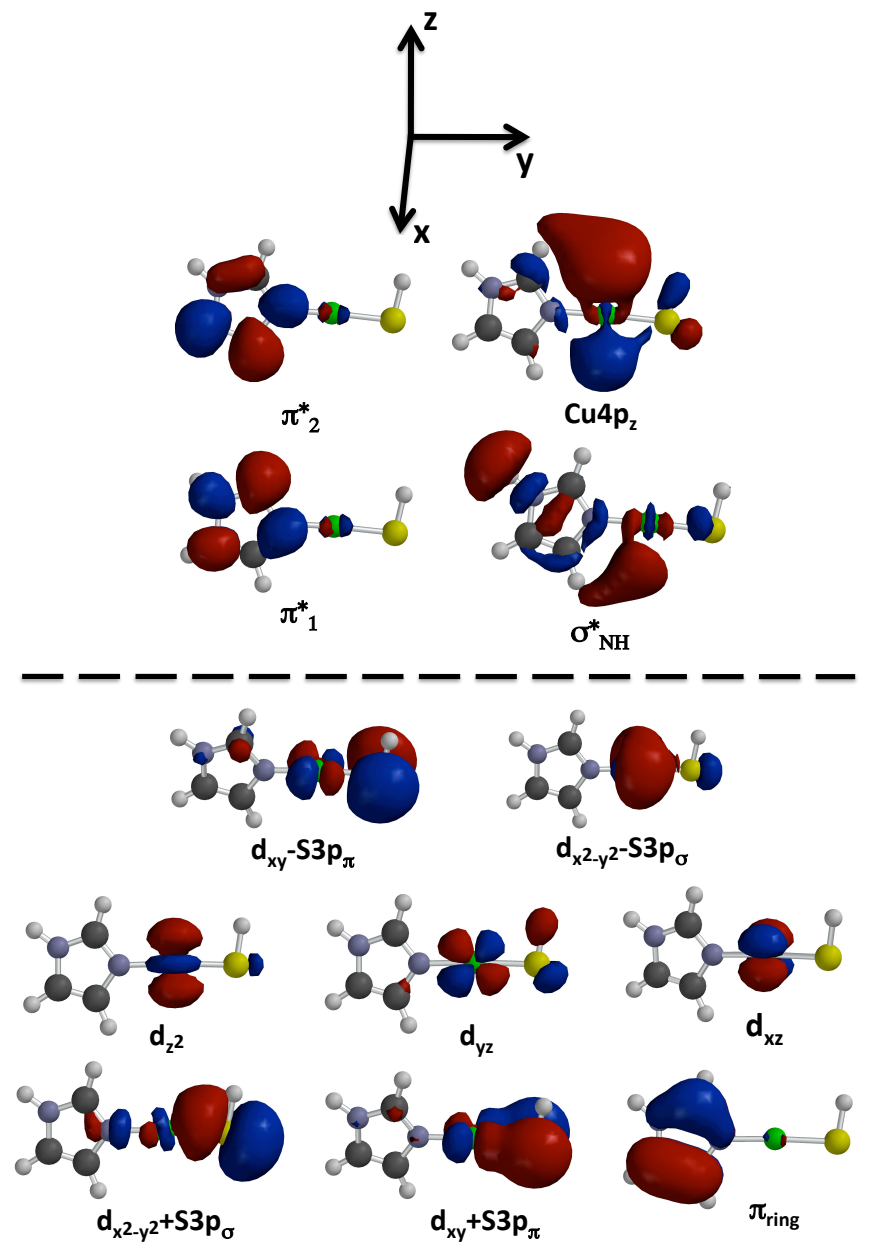


Figure 7

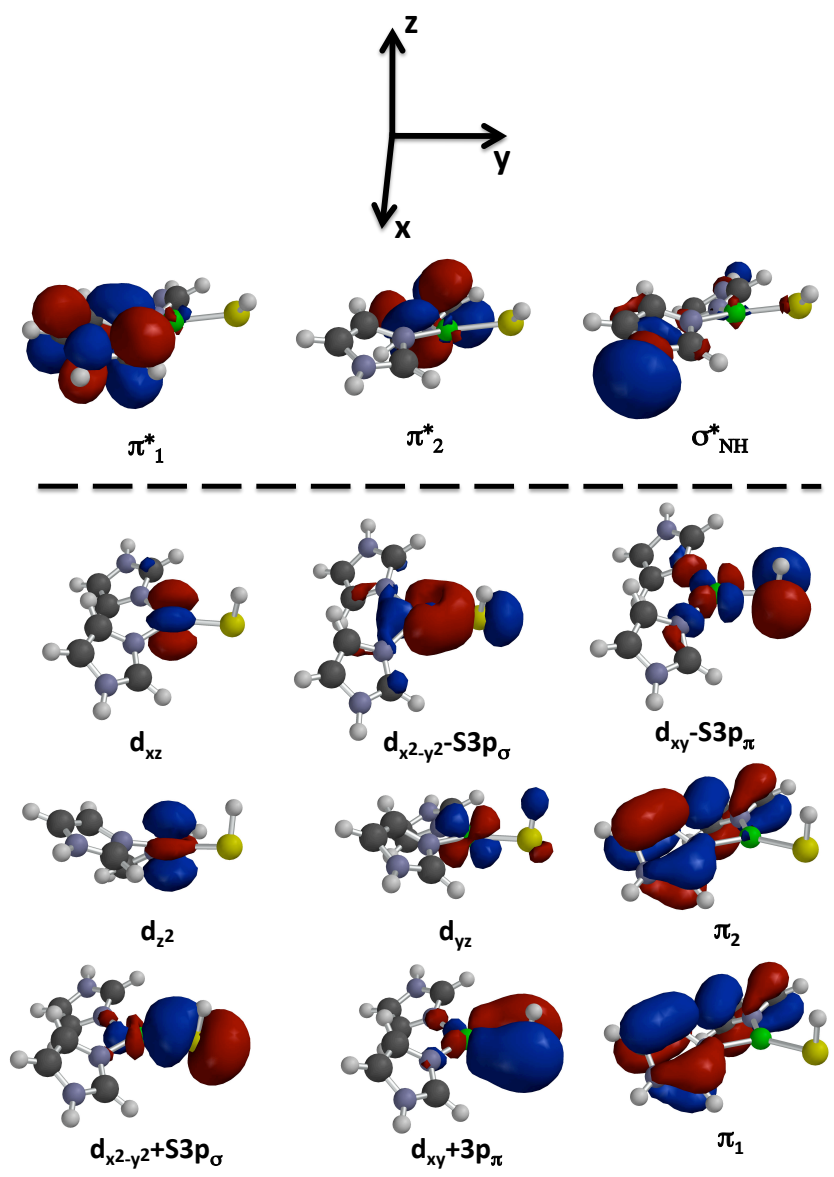


Table of Contents Graphic

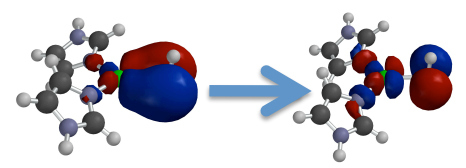

